salts, the fluorescent calcium channel ligand BODIPY FL verapamil, and the salts of the calcium indicators Fura Red, Fluo-4, and Oregon Green BAPTA-1. It should be noted that measuring solvents like methanol with a pipette can cause slightly imprecise volumes because of the high vapor pressure. In general, this method can also be used to prepare aliquots of drugs like receptor ligands or ion channel blockers.

\section{Water Recirculator (Chiller) Maintenance $^{1}$ Owen Mills}

Michigan Technological Institute opmills@mtu.edu

In my mind two things commonly cause problems for electron microscopes. One is vacuum, and the other is cooling water. It is really not that they are trouble, but rather that extra maintenance is involved. The maintenance of water recirculating systems will be discussed in this note.

A water recirculator is really a heat exchanger that operates on a basic heat transfer principle. The process cooling water within the recirculating system is forced through the scope by a pump. Heat is transferred to the process cooling water as it flows through the microscope lenses, power supplies, and diffusion pump. The process cooling water then flows back through a heat exchanger inside the water recirculator. The total heat is transferred through this heat exchanger into one of two cooling sources:

1. Refrigerated cooling system (evaporator)

2. Non-refrigerated cooling system (water-to-water heat exchanger)

In a refrigerated water recirculating system, the total heat (heat from the microscope plus heat from the mechanical compressor) is transferred through the evaporator by refrigerant into a second heat exchanger known as a condenser. A condenser may be air cooled or it may be water cooled. An air cooled condenser will transfer the total heat into the surrounding ambient air. A water cooled condenser will transfer the total heat into an in-house source of cooling water. The typical temperature range for the condenser water circuit is $50-85^{\circ} \mathrm{F}$.

In a non-refrigerated water recirculating system, the total heat is transferred through the water-to-water heat exchanger directly into an in-house source of cooling water. The typical temperature range for the water-to-water heat exchanger circuit is $40-55^{\circ} \mathrm{F}$.

Concerning maintenance, the tank water is checked weekly to insure a proper operating level. It is also a good idea to check the $\mathrm{pH}$ level of the water to make sure that it remains neutral. When it becomes dirty, it is drained and refilled with clean water. This occurs about every two years. After the system is drained, the intake filter is cleaned or replaced. A large ultrasonic cleaner can be used to clean the intake filters. Since the intake is directly before the pump, and in order to prevent pump failure, the water pump should always be primed by manually filling the intake hose before installing the filter. Distilled water is used in all systems, and mineral buildup has not been a problem. Except for one scope, all the supply hoses are opaque. Algae problems in that scope chiller are treated with Chloramine $T$ (Sigma Chemical), which effectively kills it.

The pumps used on all recirculators usually are connected to their motors via a drive key or pump coupling. This item wears under some conditions, and when it does, debris typically will be found beneath the motor and pump connection. It is a good idea to have a spare motor and pump on hand. In my experience, pumps may last 4 years or more. Few problems have been experienced with the motors themselves. Often, repair shops specialize in the repair of electrical motors. The repair can be made for less than the cost of a new motor.

Maintenance of the refrigeration systems is beyond our means with the rigorous control of refrigerants. There are sight glasses on the systems that can be used to monitor the refrigerant level. After the refrigeration compressor has been running a few minutes, bubbles in the sight glass usually indicate a low refrigerant charge. Contact a competent refrigeration specialist to check for leaks and refill if needed. When using an air-cooled condenser as mentioned above, the scope and chiller should be shut down twice a year, or more depending on the amount of dust in the area, and the condenser coils vacuumed. A restricted condenser coil will impede airflow and prevent proper heat transfer. The other heat transfer methods described previously are dependent on adequate flow of in-house cooling water to the system. Flow indicators are mounted on these lines so it is possible to detect instantly when a problem occurs. With a little extra maintenance, water recirculators will not leave us out of business.

1. Thanks are expressed to Steve Szot of the Haskris Company, for his input on this article.

\section{EDS Spectral Artifacts / Sum Peaks: A Reminder \\ Steven S. Hurban}

Endicott Interconnect Technologies, Inc.

Failure Analysis Laboratory, Endicott, NY shurban@eitny.com

Every once in a while we get involved in an analysis that brings back earlier learned lessons. Here is an example of one that proves theory can be seen in practice. It could prove helpful to new SEM/EDS analysts and also as a reminder to the more experienced.

We were looking for low levels of tin in a silicon dioxide (SiO2) matrix and having great difficulty detecting any tin level. In an attempt to stimulate more X-ray information the EDS detector count rate was increased (larger SEM spot size). This resulted in a small energy peak in the $3.4 \mathrm{KeV}$ range. We know that the Sn-La peak should be located at 3.444keV. In haste, the peak we observed could have been mislabeled either automatically using peak ID routines or manually, as the Sn-La peak we were trying to identify. However, closer examination of the exact peak location revealed that this peak centroid did not line up at the $3.444 \mathrm{keV}$ mark. The peak was obviously at a slightly higher energy than where we would expect to see the $S n-L a$ peak. Also, any secondary peaking associated with the Sn-La peak was absent. A Si-Ka SUM peak would have a centroid of $2 x$ the SiKa energy $(1.740 \mathrm{keV})$, or $3.480 \mathrm{keV}$. The peak centroid lined up perfectly with this energy value. The Sn-La / Si-Ka SUM peak overlap is a classic example of this artifact.

Increasing the count rate to $>15,000$ counts per second resulted in approximately $60 \%$ dead time - not optimum EDS acquisition parameters 1 These instrument parameters can not be overlooked when collecting spectral data. Analyzing the same $\mathrm{SiO}_{2}$ rich areas using different instrument parameters ( $<3000 \mathrm{CPS}$, approx. $20 \%$ dead time), we were able to eliminate the artifactual peak at this location. This was supportive proof that the peak was indeed a Si-Ka SUM peak.

This analysis served as a reminder that there are specific instrument specimen conditions that will enhance the occurrence of spectral artifacts. We were also reminded to investigate suspicious AUTO ID results. Knowledge of both the sample and the instrumentation capabilities/ limitations play an integral role in accurate and complete elemental analysis. Even today's systems, that incorporate an efficient pulse pileup rejector, can not separate two pulses closely spaced in time. Overloading the EDS detector, limits it's capability to separate pulses in nearly exact coincidence and results in the formation of a small pulse pileup peak in the EDS spectrum. ${ }^{2,3}$

\section{References}

1) J.I. Goldstein, D.E. Newbury, P. Echlin, D.C. Joy, C. Fiori, E. Lifshin, Scanning Electron Microscopy and Microanalysis, New York, Plenum Press (1992) 275-292

2) J.I. Goldstein, D.E. Newbury, P. Echlin, D.C. Joy, C. Fiori, E. Lifshin, Scanning Electron Microscopy and Microanalysis, New York, Plenum Press (1992) 226-241

3) C.E. Fiori, D.E. Newbury, R.L. Myklebust, Artifacts Observed in Energy Dispersive X-Ray Spectrometry in Electron Beam Instruments - A Cautionary Guide, NBS Special Publication 604 (1981) 315-333.

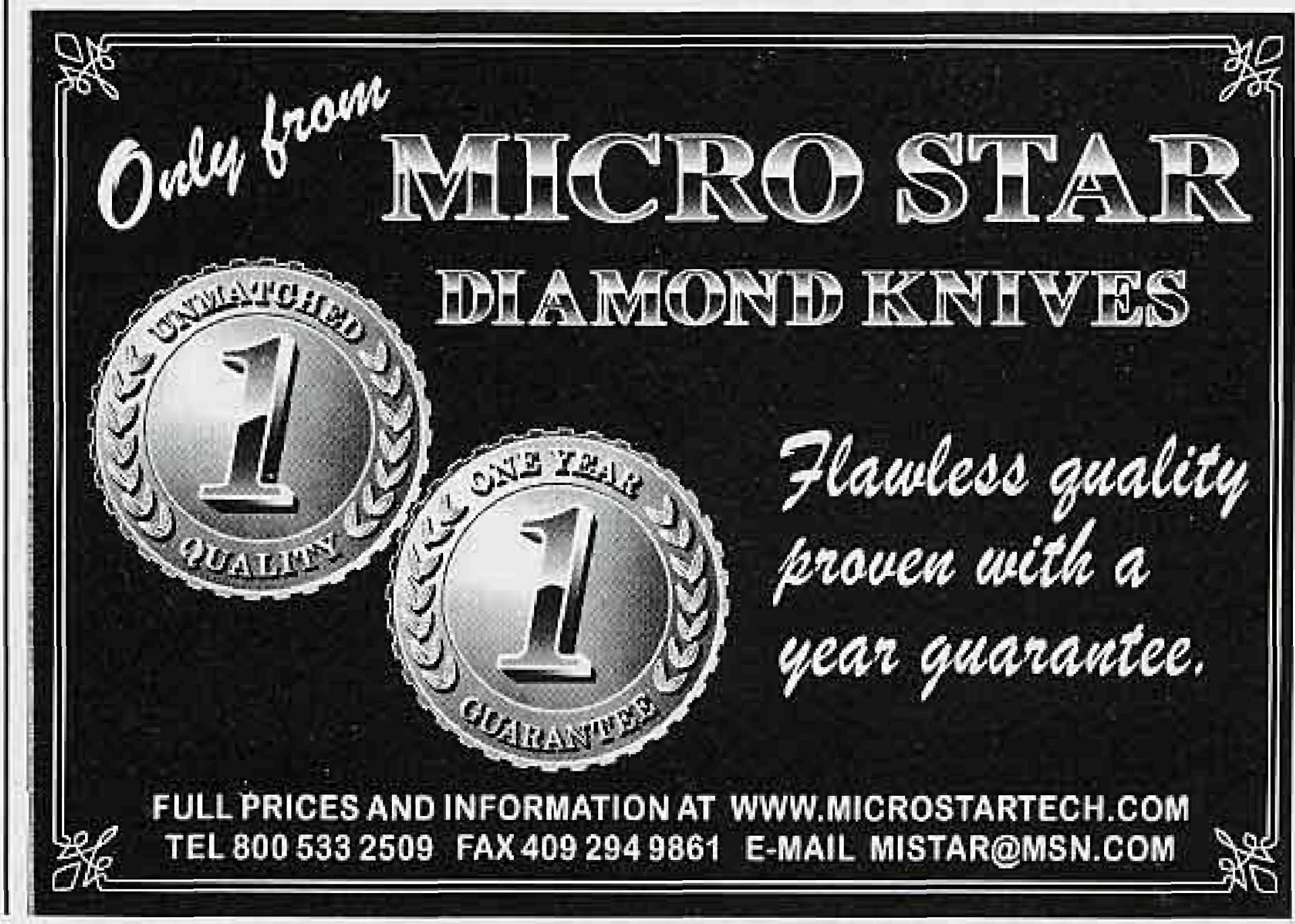

